\title{
Ethylene Polymerization by Novel, Easily Accessible Catalysts Based on Nickel(II) Diazene Complexes
}

\section{Supporting Information}

\section{Dirk L. Schröder, Wilhelm Keim, Stefan Mecking}

General Methods and Materials. Nickel(II) complexes were manipulated by standard Schlenk techniques. Argon was purified by passage through columns of BASF R311 catalyst and $4 \AA$ molecular sieves. Ethylene (99.8\%) supplied by Gerling and Holz was used without further purification. Organic solvents were distilled from drying agents (dichloromethane: $\mathrm{CaH}_{2}$; pentane: $\mathrm{KOH}$; toluene: $\mathrm{Na}$ ) under Argon. Deionized water was degassed prior to use. [(tmeda)Ni( $\left.\left.\mathrm{CH}_{3}\right)_{2}\right]^{1}$ was purchased from MCAT GmbH (Konstanz, Germany) and used as received. IR spectra were recorded on a Nicolet 510 P FT spectrometer. Elemental analyses were performed on a Carlo Erba CHN 1106 instrument. ${ }^{1} \mathrm{H}$ and ${ }^{13} \mathrm{C}$ NMR spectra were recorded on a Bruker DPX 300 instrument at 300 and $75 \mathrm{MHz}$, respectively. ${ }^{1} \mathrm{H}$ and ${ }^{13} \mathrm{C}$ NMR spectra of polyethylenes were obtained in 1,1,2,2-tetrachloroethane- $d_{2}$ at $122{ }^{\circ} \mathrm{C}$. The branching structure was assigned according to [2]. Differential scanning calorimetry (DSC) was performed on a Perkin-Elmer DSC 7 or on a Pyris 1 DSC at a heating rate of $10 \mathrm{~K} / \mathrm{min}$. DSC data reported are second heats. Polymer crystallinities were calculated based on a melt enthalpy of $293 \mathrm{~J} \mathrm{~g}^{-1}$ for $100 \%$ crystalline polyethylene. High-temperature gel permeation chromatography was performed in $1,2,4$-trichlorbenzene at $140{ }^{\circ} \mathrm{C}$ using a PL-220 instrument equipped with Mixed Bed PL-columns. Data reported were referenced to linear polyethylene standards (universal calibration). Gas chromatographic analysis of ethylene 
oligomers was performed on a Siemens Sichromat equipped with a $25 \mathrm{~m}$ FS-SE-54-cs-0,35 column.

Synthesis of 1a-c. The ligands were prepared by the coupling reaction of a diazonium salt with 2-naphthol at $0{ }^{\circ} \mathrm{C}$. The diazonium salt was generated by reacting the arylamine (20 mmol) with isoamylnitrite $(25 \mathrm{mmol})$ and $\mathrm{BF}_{3} \cdot \mathrm{OEt}_{2}(22 \mathrm{mmol})$ in dichloromethane as a solvent $(200 \mathrm{~mL})$ at $-10^{\circ} \mathrm{C}$ for $15 \mathrm{~min}$. A pink precipitate formed rapidly. After brief isolation by filtration at low temperature it was dissolved in basic aqueous solution $(100 \mathrm{~mL}$ of $10 \%$ $\mathrm{NaOH}$ ) and immediately reacted with one equivalent of 2-naphthol at $0{ }^{\circ} \mathrm{C}$, stirring for $1 \mathrm{~h}$. The diazen-naphthol obtained was recrystallized from toluene/ethanol to afford red crystals. 1c is also commercially available as an azo dye (Sudan I, C.I. 12055). 1a (41\% yield): ${ }^{1} \mathrm{H}-$ $\operatorname{NMR}\left(\mathrm{CDCl}_{3}\right): \delta=1.28\left(\mathrm{~d},{ }^{3} \mathrm{~J}=7 \mathrm{~Hz}, 12 \mathrm{H}, \mathrm{CH}_{3}{ }^{\mathrm{PPr}}\right.$ ), 3.34 (septett, ${ }^{3} \mathrm{~J}=7 \mathrm{~Hz}, 2 \mathrm{H}, \mathrm{CH}^{\mathrm{iPr}}$ ), $6.95(\mathrm{~d}$, $\left.1 \mathrm{H}, \mathrm{H}_{\text {arom }}\right), 7.2-7.4\left(\mathrm{~m}, 4 \mathrm{H}, \mathrm{H}_{\text {arom }}\right), 7.50\left(\mathrm{t}, 1 \mathrm{H}, \mathrm{H}_{\text {arom }}\right), 7.61\left(\mathrm{~d}, 1 \mathrm{H}, \mathrm{H}_{\text {arom }}\right), 7.74\left(\mathrm{~d}, 1 \mathrm{H}, \mathrm{H}_{\text {arom }}\right)$, $8.43\left(\mathrm{~d}, 1 \mathrm{H}, \mathrm{H}_{\text {arom }}\right), 16.22(\mathrm{~s}, 1 \mathrm{H}, \mathrm{OH}) ;{ }^{13} \mathrm{C}\left\{{ }^{1} \mathrm{H}\right\}-\mathrm{NMR}\left(\mathrm{CDCl}_{3}\right): \delta=23.8,28.2,121.3,123.9$, $124.6,125.3,127.8,128.2,128.5,128.8,130.5,133.7,139.6,140.5,142.2,169.8 ;$ Anal. Calcd for $\mathrm{C}_{22} \mathrm{H}_{24} \mathrm{~N}_{2} \mathrm{O}$ : C 79.48; $\mathrm{H} 7.28 ; \mathrm{N} 8.43$. Found: $\mathrm{C} 79.51 ; \mathrm{H} 7.39 ; \mathrm{N} 8.35 .1 \mathrm{~b}\left(68 \%\right.$ yield): ${ }^{1} \mathrm{H}-$ $\operatorname{NMR}\left(\mathrm{CDCl}_{3}\right): \delta=2.65\left(\mathrm{~s}, 6 \mathrm{H}, \mathrm{CH}_{3}\right), 6.93\left(\mathrm{~d}, 1 \mathrm{H}, \mathrm{H}_{\text {arom }}\right), 7.16-7.43\left(\mathrm{~m}, 3 \mathrm{H}, \mathrm{H}_{\text {arom }}\right), 7.54(\mathrm{t}$, $\left.1 \mathrm{H}, \mathrm{H}_{\text {arom }}\right), 7.57\left(\mathrm{t}, 1 \mathrm{H}, \mathrm{H}_{\text {arom }}\right), 7.64\left(\mathrm{~d}, 1 \mathrm{H}, \mathrm{H}_{\text {arom }}\right), 7.75\left(\mathrm{~d}, 1 \mathrm{H}, \mathrm{H}_{\text {arom }}\right), 8.43\left(\mathrm{~d}, 1 \mathrm{H}, \mathrm{H}_{\text {arom }}\right)$, $16.18(\mathrm{~s}, 1 \mathrm{H}, \mathrm{OH}) ;{ }^{13} \mathrm{C}\left\{{ }^{1} \mathrm{H}\right\}-\mathrm{NMR}\left(\mathrm{CDCl}_{3}\right): \delta=20.4,121.3125 .1,125.3,126.8,127.8,128.6$, $128.9,129.8,130.1,130.7,133.7,139.7,140.7,172.0 ;$ Anal. Calcd for $\mathrm{C}_{18} \mathrm{H}_{16} \mathrm{~N}_{2} \mathrm{O}: \mathrm{C} 78.23 ; \mathrm{H}$ 5.84; N 10.14. Found: C 78.18; $\mathrm{H} 5.78 ; \mathrm{N} 10.21$. 1c (90\% yield): ${ }^{1} \mathrm{H}-\mathrm{NMR}\left(\mathrm{CDCl}_{3}\right): \delta=6.7-$ $8.4\left(\mathrm{~m}, 11 \mathrm{H}, \mathrm{H}_{\text {arom }}\right), 16.1(\mathrm{~s}, 1 \mathrm{H}, \mathrm{OH}) ;{ }^{13} \mathrm{C}\left\{{ }^{1} \mathrm{H}\right\}-\mathrm{NMR}\left(\mathrm{CDCl}_{3}\right): \delta=115.6,118.3,119.0,122.2$, $125.3,126.2,127.8,128.5,129.0,129.3,129.8,130.0,134.2,140.6,145.0,172.7$; Anal. Calcd for $\mathrm{C}_{16} \mathrm{H}_{12} \mathrm{~N}_{2} \mathrm{O}$ : C 77.40; H 4.87; N 11.29. Found: C 77.14; H 4.97; N 11.26. 
Synthesis of complexes $\left[\left(\mathrm{N}=\mathrm{N}^{\wedge} \mathbf{O}\right) \mathrm{Ni}(\mathrm{Me})\right.$ (pyridine)] (2a-c). The novel complexes

2a-c were obtained by reaction of the diazenenaphthols 1a-c with 1 equiv of [(tmeda) $\left.\mathrm{Ni}\left(\mathrm{CH}_{3}\right)_{2}\right]$ in the presence of a 10 -fold excess of pyridine in diethyl ether as a solvent at $-40{ }^{\circ} \mathrm{C}$. After stirring for $2 \mathrm{~h}$ at $0{ }^{\circ} \mathrm{C}$ the red solution was filtered through a frit with dry Celite, and the solvent was removed under reduced pressure. Yields were virtually quantitative. 2a: ${ }^{1} \mathrm{H}-\mathrm{NMR}\left(\mathrm{C}_{6} \mathrm{D}_{6}\right): \delta-0.29\left(\mathrm{~s}, 3 \mathrm{H}, \mathrm{Ni}-\mathrm{CH}_{3}\right), 1.15$ and $1.56,\left(\mathrm{~d},{ }^{3} \mathrm{~J}=7 \mathrm{~Hz}\right.$, $\left.2 \times 6 \mathrm{H}, \mathrm{CH}_{3}{ }^{\mathrm{iP}}\right), 4.23$ (septett, $\left.{ }^{3} \mathrm{~J}=7 \mathrm{~Hz}, 2 \mathrm{H}, \mathrm{CH}{ }^{\mathrm{iPr}}\right), 6.5-8.7\left(14 \mathrm{H}, \mathrm{H}_{\text {arom }}\right) ;{ }^{13} \mathrm{C}\left\{{ }^{1} \mathrm{H}\right\}-\mathrm{NMR}$ $\left(\mathrm{C}_{6} \mathrm{D}_{6}\right): \delta-5.9\left(\mathrm{Ni}_{-} \mathrm{CH}_{3}\right), 22.4,22.7,27.1,120.1,122.6,122.8,126.4,126.6,126.9,127.1$, $127.2,127.3,127.6,127.8,130.5,137.5,140.7,141.6,147.8 .2 \mathrm{~b}:{ }^{1} \mathrm{H}-\mathrm{NMR}\left(\mathrm{C}_{6} \mathrm{D}_{6}\right): \delta-0.28$ (s, $\left.3 \mathrm{H}, \mathrm{Ni}-\mathrm{CH}_{3}\right), 2.69\left(\mathrm{~s}, 6 \mathrm{H}, \mathrm{CH}_{3}\right), 6.7-8.6\left(14 \mathrm{H}, \mathrm{H}_{\text {arom }}\right) ;{ }^{13} \mathrm{C}\left\{{ }^{1} \mathrm{H}\right\}-\mathrm{NMR}\left(\mathrm{C}_{6} \mathrm{D}_{6}\right): \delta \delta=-5.6(\mathrm{Ni}-$ $\left.\mathrm{CH}_{3}\right), 20.8,119.3,120.9,121.1,122.1,122.8,125.5,126.7,128.8,129.0,129.4,129.8,130.2$, $132.7,133.2,141.2,141.8 .2 \mathrm{c}:{ }^{1} \mathrm{H}-\mathrm{NMR}\left(\mathrm{C}_{6} \mathrm{D}_{6}\right): \delta-0.26\left(\mathrm{~s}, 3 \mathrm{H}, \mathrm{Ni}-\mathrm{CH}_{3}\right), 6.6-8.8(16 \mathrm{H}$, $\left.\mathrm{H}_{\text {arom }}\right) ;{ }^{13} \mathrm{C}\left\{{ }^{1} \mathrm{H}\right\}-\mathrm{NMR}\left(\mathrm{C}_{6} \mathrm{D}_{6}\right): \delta-5.6\left(\mathrm{Ni}-\mathrm{CH}_{3}\right), 115.7,118.5,119.2,122.4,125.5,126.4$, $127.9,128.8,129.2,129.4,129.9,130.2,133.3,134.2,140.9,145.4$. Due to the sensitivity of the compounds, meaningful elemental analysis could not be obtained.

Polymerization/Oligomerization Procedure. Polymerization was carried out in a 75 $\mathrm{mL}$ steel reactor, equipped with a magnetic stirr bar. The reactor was repeatedly evacuated and backfilled with nitrogen. A solution of the catalyst precursor (complex 2) in $20 \mathrm{~mL}$ toluene was cannula-transferred to the reactor. The reactor was flushed with ethylene, and a constant ethylene pressure was applied as the vessel was rapidly heated to the specified temperature by means of an oil bath. After the specified reaction time, the reaction was stopped by cooling and releasing the ethylene pressure. Precipitated polymers were isolated by filtration, washed with methanol, and dried in vacuum prior to further analysis. The filtrate 
was analyzed by gas chromatography.

(1) Kaschube, W.; Poerschke, K. R.; Wilke, G.J. Organomet. Chem. 1988, 355, 525-32.

(2) a) Randall, J. C.J. Macromol. Sci., Rev. Macromol. Chem. Phys. 1989, C29, $201-317$. b) Axelson, D. E.; Levy, G. C.; Mandelkern, L. Macromolecules 1979, 12, 41 - 52.

(3) a) Nölting, E.; Kohn, O. Chem. Ber. 1884, 17, 351-69. b) Laali, K.; Szele, I.; Zollinger, H. Helv. Chim. Acta 1983, 66, 1737-47. c) Encylopedia of Reagents for Organic Synthesis, Ed. Paquette, L. A.; John Wiley, Chichester, 1983, Vol. 4, p. 2908-11. 


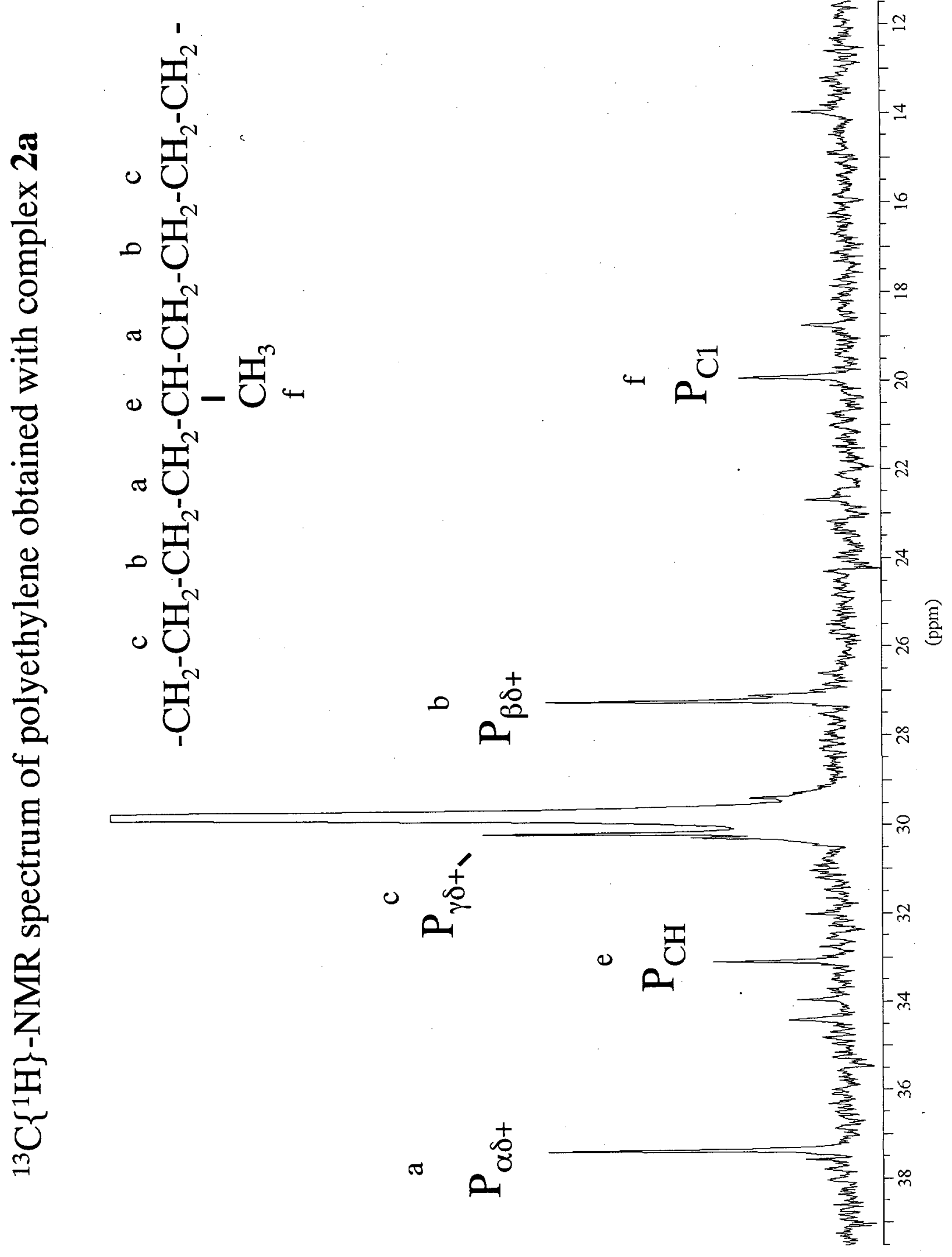

